\title{
A Genetically Encoded AND Gate for Cell-Targeted Metabolic Labeling of Proteins
}

\author{
Alborz Mahdavi, ${ }^{\dagger}{ }^{\dagger}$ Thomas H. Segall-Shapiro, ${ }^{\S, \perp, \infty}$ Songzi Kou, $^{\dagger}$ Granton A. Jindal, ${ }^{\dagger, \text { Il }}$ Kevin G. Hoff, ${ }^{\S, \#}$ \\ Shirley Liu, ${ }^{\S}$ Mohsen Chitsaz, ${ }^{\|}$Rustem F. Ismagilov, ${ }^{\dagger}$ Jonathan J. Silberg, ${ }^{\S, \perp}$ and David A. Tirrell ${ }^{*}, \dagger,+$ \\ ${ }^{\dagger}$ Division of Chemistry and Chemical Engineering, ${ }^{\ddagger}$ Bioengineering, and "Biochemistry and Molecular Biophysics, California Institute \\ of Technology, Pasadena, California 91125, United States \\ ${ }^{\S}$ Department of Biochemistry and Cell Biology and ${ }^{\perp}$ Department of Bioengineering, Rice University, Houston, Texas 77251 , United \\ States
}

ABSTRACT: We describe a genetic AND gate for celltargeted metabolic labeling and proteomic analysis in complex cellular systems. The centerpiece of the AND gate is a bisected methionyl-tRNA synthetase (MetRS) that charges the Met surrogate azidonorleucine (Anl) to tRNA $^{\text {Met }}$. Cellular protein labeling occurs only upon activation of two different promoters that drive expression of the $\mathrm{N}$ - and C-terminal fragments of the bisected MetRS. Anl-labeled proteins can be tagged with fluorescent dyes or affinity reagents via either copper-catalyzed or strainpromoted azide-alkyne cycloaddition. Protein labeling is apparent within 5 min after addition of Anl to bacterial cells in which the AND gate has been activated. This method allows spatial and temporal control of proteomic labeling and identification of proteins made in specific cellular subpopulations. The approach is demonstrated by selective labeling of proteins in bacterial cells immobilized in the center of a laminar-flow microfluidic channel, where they are exposed to overlapping, opposed gradients of inducers of the $\mathrm{N}$ - and C-terminal MetRS fragments. The observed labeling profile is predicted accurately from the strengths of the individual input signals.

$\mathrm{T}$ he bio-orthogonal noncanonical amino acid tagging (BONCAT) method of proteomic analysis enables enrichment and identification of selected subsets of cellular proteins. ${ }^{1}$ The initial demonstration of the BONCAT method used the methionine (Met) surrogate azidohomoalanine (Aha) as a metabolic label; addition of Aha to cells at selected time points provides temporal control of proteomic labeling. In contrast to Aha, which is incorporated into proteins in all cells through the action of the wild-type methionyl-tRNA synthetase (MetRS), the bulkier Met surrogate azidonorleucine (Anl) can be used to restrict labeling to cells that express a mutant MetRS (designated NLL-MetRS). ${ }^{2}$ Sensitivity to cell state (e.g., to oxidative stress) can be achieved by controlling expression of the mutant synthetase with carefully chosen promoters. ${ }^{3}$ However, it is not yet possible to specify cell state more precisely in such experiments, and to control proteomic labeling by integrating multiple signals to activate the NLLMetRS.
Here we introduce a new approach in which multiple promoters ( $\mathrm{P} 1$ and $\mathrm{P} 2)$ are used to regulate Anl incorporation into the E. coli proteome. The approach relies on construction of a split MetRS that generates a translational output only in cells in which the P1 and P2 inputs are simultaneously on. The split MetRS functions as an AND gate to control protein labeling (Figure 1).

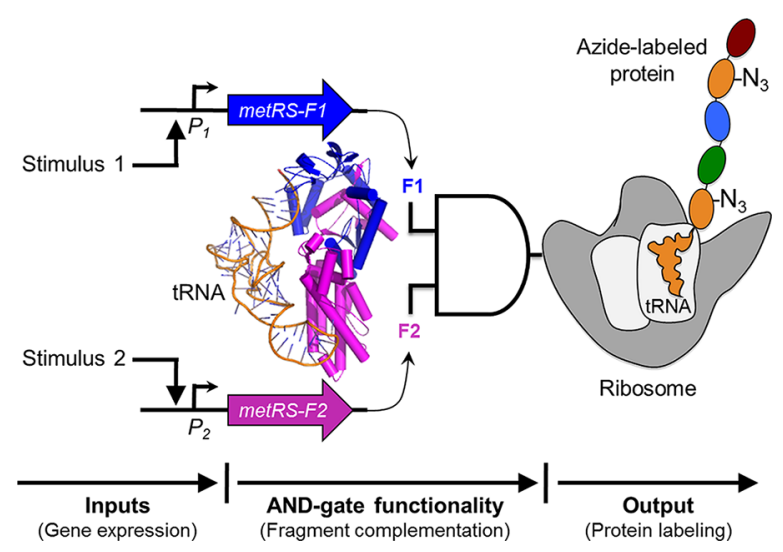

Figure 1. A genetic AND gate for proteomic labeling. Two promoters (P1 and P2) are used as inputs to drive expression of E. coli MetRS fragments F1 and F2. tRNA ${ }^{\text {Met }}$ is charged with Anl only when the MetRS fragments are both expressed, and associate to form a functional enzyme. Ribosomes use Anl-charged tRNA ${ }^{\text {Met }}$ during protein synthesis in competition with Met-charged tRNA ${ }^{\text {Met }}$. Newly synthesized proteins are labeled with azide groups, which can be selectively tagged by the azide-alkyne cycloaddition. ${ }^{4}$ Diverse promoter inputs can be plugged into the AND gate to control proteomic labeling with Anl. The MetRS image was created using PDB $2 \mathrm{CSX}^{5}$

We used protein fragment complementation to make a bisected MetRS, ${ }^{6}$ prompted by the work of Schimmel and coworkers, who showed that the E. coli MetRS could be cut at six different sites to yield functional split variants. ${ }^{7}$ To assess which of the peptide bonds in the MetRS backbone should be broken to create fragments that associate to form the most active enzymes, transposase mutagenesis was used to construct a

Received: January 15, 2013

Published: February 13, 2013 
library of vectors that express split MetRS, ${ }^{8}$ and active variants were selected via bacterial complementation. To avoid variants that require only a single fragment for activity, a gene fragment encoding MetRS residues 1-548 (designated MetRS henceforth) was used to construct the vector library (Figure 2a). The 1-548 variant is the smallest MetRS fragment that has been reported to exhibit near-native activity and stability. ${ }^{9}$ E. coli strain CS50 was used for selecting functional variants on medium lacking Met; ${ }^{10}$ growth was visible only when cells expressed an active MetRS (Figure 2b).

Selection by CS50-DE3 complementation yielded hundreds of colonies with varying complementation strengths (Figure S1). DNA sequencing of these clones identified MetRS variants with backbone cleavage sites lying within all four domains of the synthetase (Figure 2c, Table S1). Variants with the greatest complementation strength were cut within the connective polypeptide and Rossmann domains. Mapping the locations of backbone cleavage onto the MetRS structure (Figure 2d) revealed that cleavage in these domains yields fragments that make large numbers of intermolecular residue-residue contacts in the assembled enzyme (Figure S2).

We have shown previously that two MetRS triple mutants, L13N/Y260/H301 (NLL) and L13P/Y260/H301 (PLL), charge Anl with good efficiency. ${ }^{12}$ The corresponding mutations were introduced into the vectors encoding the bisected MetRS variants that exhibited the strongest complementation (those with cut sites at residues 48, 131, 183, 247, 272, 278, and 456) (Figure S3). E. coli CS50-DE3 cells transformed with each mutant vector were grown to midlog phase in liquid culture, and labeling was initiated by addition of $1 \mathrm{mM} \mathrm{Anl} \mathrm{(1,} \mathrm{Figure} \mathrm{2e).} \mathrm{After} 1 \mathrm{~h}$, cells were lysed, and total cellular proteins were treated with alkynetetramethylrhodamine (TAMRA) dye (2, Figure 2e) for labeling via copper-catalyzed azide-alkyne cycloaddition (3, Figure 2e). ${ }^{4,13,14}$ After SDS-PAGE, TAMRA-labeled proteins were detected by in-gel fluorescence imaging (Figure 2f). All 14 of the NLL and PLL split MetRS variants enabled Anl incorporation into cellular proteins. On the basis of this result and the strong complementation observed for wild-type MetRS(247) (Figure 2b), NLL-MetRS(247) was selected for further development.

To construct an AND gate for controlled labeling of cellular proteins with Anl, the coding sequences for the $\mathrm{N}$ - and $\mathrm{C}$ terminal fragments of NLL-MetRS(247) were placed in a pair of vectors under control of $\mathrm{P}_{\mathrm{T} 5}$ and $\mathrm{P}_{\mathrm{BAD}}$ promoters, respectively. As expected, NLL-MetRS(247) showed IPTGand arabinose-dependent activity, with the highest levels of metabolic protein labeling observed in cultures supplemented with both inducers. To be useful as an AND gate, the system described here should be active only when both IPTG and arabinose are present-not in the presence of a single inducer. However, we observed some activity even in the absence of IPTG, presumably as a consequence of leaky expression of the $\mathrm{N}$-terminal fragment (Figure S4). This activity was readily eliminated by catabolite repression (Figure S5); ${ }^{15}$ in glucosesupplemented media, Anl labeling was observed only upon addition of both IPTG and arabinose (Figure $3 a-c$ ).

To investigate how the output of the AND gate was controlled by variation in input levels, we used varying combinations of arabinose and IPTG concentrations to induce MetRS fragment expression and Anl incorporation. We found concentration-dependent expression of the $\mathrm{N}$ - and C-terminal fragments (Figure S6) and steep transitions between the on and
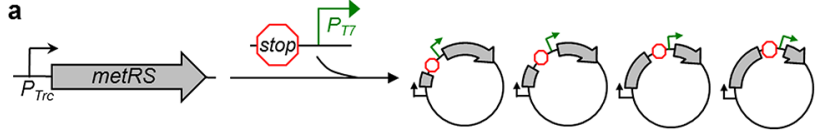

b
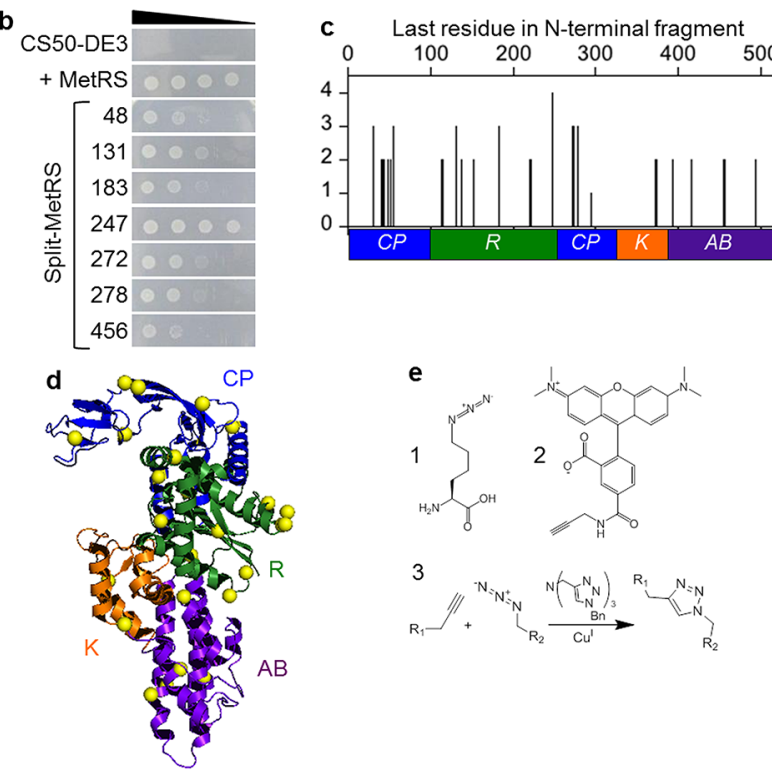

3
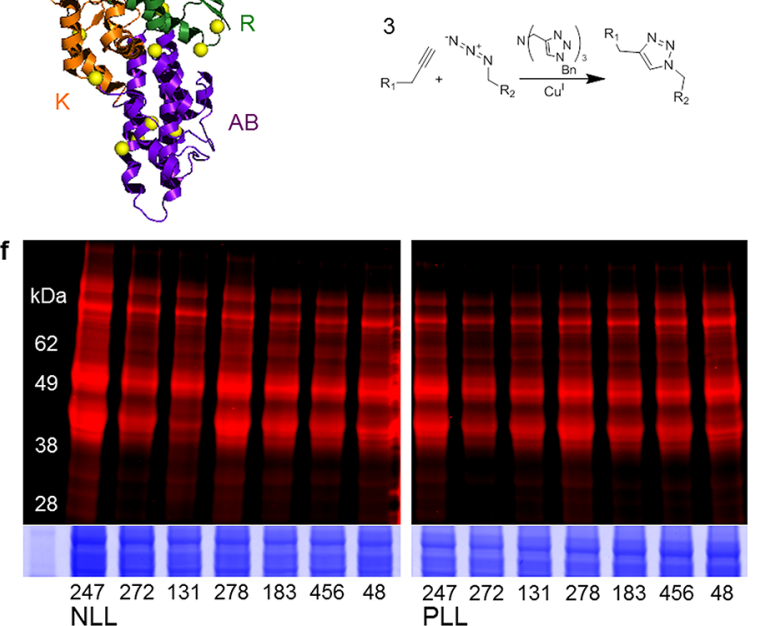

Figure 2. Identifying fragmented MetRS variants that metabolically label proteins with Anl. (a) DNA that contains a terminator, promoter $\left(\mathrm{P}_{\mathrm{T} 7}\right)$, and ribosomal binding site was randomly inserted into the MetRS gene to create a library of vectors that express two-piece MetRS variants. ${ }^{8}$ (b) Growth of E. coli CS50-DE3 cells in the absence of Met is compared for cells that express a functional MetRS truncation comprised of residues $1-548$ and split MetRS variants. Ten-fold serial dilutions of cells were spotted on M9-agar plates and growth was imaged after $24 \mathrm{~h}$. (c) Complementation strengths of fragmented MetRS variants, which were named based on the last residue encoded by their $\mathrm{N}$-terminal fragments. (d) Color coding of the connective polypeptide [CP, blue], Rossmann fold [R, green], KMSKS [K, orange], and anticodon-binding $[\mathrm{AB}$, purple] domains maps the locations of backbone fission (yellow spheres) in functional variants onto the MetRS structure. ${ }^{11}$ (e) Structure of Anl (1), which can be detected in proteins after reaction with alkyne-functionalized tetramethylrhodamine (TAMRA) dye (2). Copper-catalyzed azidealkyne cycloaddition (3) results in a stable triazole linkage. (f) Cells expressing the split NLL-MetRS and PLL-MetRS from the vectors selected using bacterial complementation were grown in the presence of Anl, and cell lysates were treated with TAMRA-alkyne dye. In-gel fluorescence image shows TAMRA labeling, which indicates Anl incorporation into cellular proteins. Colloidal blue staining (bottom) was performed to compare protein loading across lanes.

off states (Figures 3d and S6). The time dependence of Anl labeling under control of the activated AND gate was monitored by detection of labeled proteins with an azadibenzocyclooctyne-TAMRA dye; labeling was apparent within $5 \mathrm{~min}$ after addition of Anl (Figure 3e). The rate of 

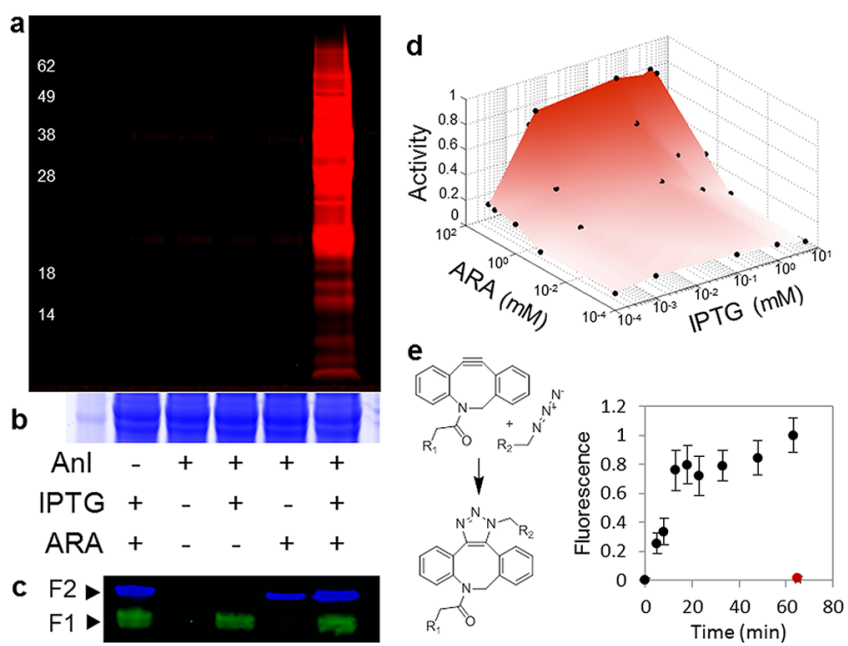

Figure 3. Genetic control of Anl incorporation into proteins in cells that express the $\mathrm{N}$ - and C-terminal fragments of NLL-MetRS(247) under control of IPTG- and arabinose-inducible promoters. (a) Anl labeling of E. coli proteins in the presence or absence of IPTG (1 $\mathrm{mM})$, arabinose $(5 \mathrm{mM})$, and Anl $(1 \mathrm{mM})$. Cell lysates were treated with an alkyne-TAMRA dye, and in-gel TAMRA fluorescence was imaged to detect Anl incorporation. (b) Colloidal blue staining of the gel in (a) confirms similar total protein in each sample. (c) Western blot detection of the $\mathrm{N}$-terminal (green) fragment containing a $\mathrm{His}_{6}$ tag and the C-terminal (blue) fragment containing a hemagglutinin tag. (d) Effects of arabinose and IPTG levels on Anl incorporation. Cell lysates were treated with alkyne-TAMRA, and the in-gel fluorescence signal from TAMRA-labeled proteins was used to determine relative Anl incorporation levels. Dots on the surface show conditions under which Anl incorporation was measured. (e) Time-dependence of protein labeling upon addition of Anl to cells harboring the activated AND gate. IPTG $(1 \mathrm{mM})$ and arabinose (5 $\mathrm{mM})$ were added to activate the AND gate; Anl $(1 \mathrm{mM})$ was added 3 $\mathrm{h}$ later (at $0 \mathrm{~min}$ on the time axis). Anl-labeled proteins were detected by treatment with azadibenzocyclooctyne-TAMRA dye (R1); labeling was quantified by in-gel fluorescence detection. When the AND gate was not activated but Anl was present, no measurable incorporation was observed even after $1 \mathrm{~h}$, as shown by the red symbol.

AND gate activation upon induction by IPTG and arabinose was also measured (Figure S7). The rate of activation will depend on the particular promoters used to drive fragment expression.

Spatially controlled proteomic labeling of cells within complex multicellular systems is useful for study of a variety of important biological phenomena including biofilm formation and developmental patterning. ${ }^{16,17}$ The MetRS AND gate provides a useful solution to such challenges because it can be activated by spatially regulated signals. As a step toward this goal, we sought to control Anl labeling in cells that are subjected to spatial gradients of activators. A laminar-flow microfluidic channel was used to expose surface-immobilized bacterial cells harboring the AND gate to opposing gradients of IPTG and arabinose. Anl incorporation should occur only in cells that encounter sufficient levels of both inducers (Figure 4a). The diffusion of IPTG and arabinose in the microfluidic channel was modeled (Figure S8), and diffusion analysis results were used to estimate inducer profiles across the width of the channel (Figure 4b).

Dye diffusion experiments enabled visualization of similar gradients during flow, and confirmed the modeling results (Figure S9). To estimate the strengths of the individual AND gate inputs, E. coli DH10B cells were transformed with
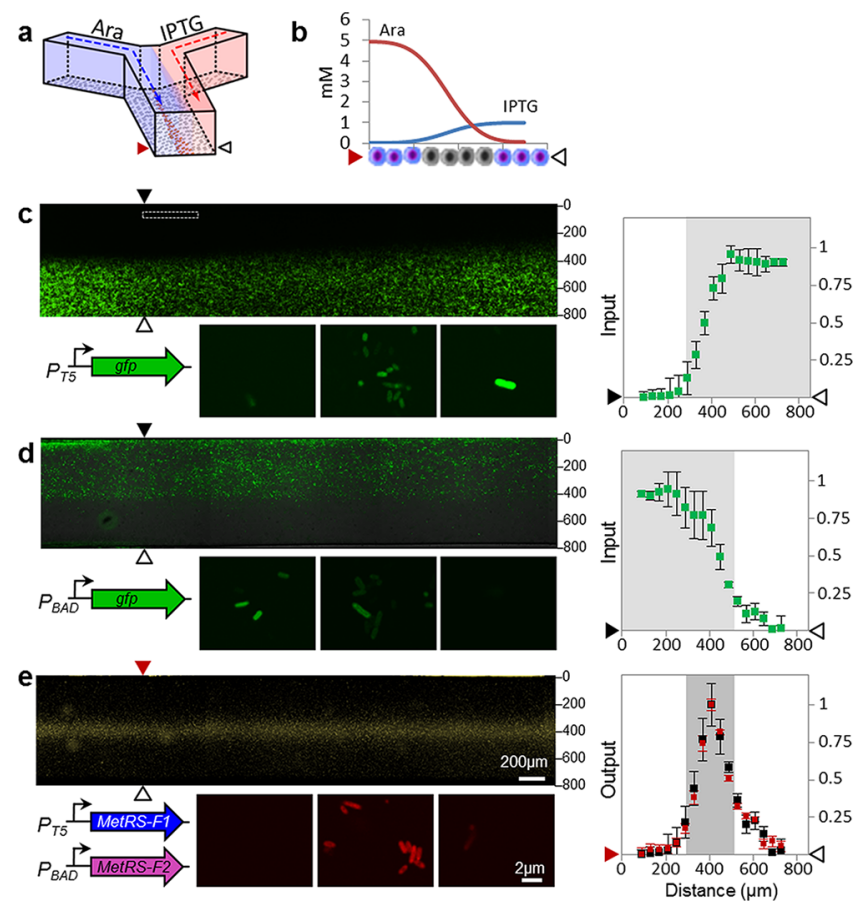

Figure 4. Spatial control of protein labeling. (a) A laminar flow microfluidic channel was used to expose surface-immobilized cells to gradients of arabinose and IPTG. In this device, the AND gate should be activated only in the center of the channel, where cells are exposed to both IPTG and arabinose. (b) Modeling of inducer diffusion was used to determine cross-channel concentration profiles of arabinose and IPTG at the locations shown by the arrows in (c, d, and e). Schematic shows that cells in the middle of the channel should incorporate Anl (gray); those on the periphery should not be labeled (purple). (c) Fluorescence image of the microfluidic channel (top) seeded with cells expressing GFP under IPTG control, and images of individual cells (bottom) that were incubated with varying combinations of inducers (left to right; $5 \mathrm{mM}$ arabinose, $1 \mathrm{mM}$ arabinose plus $0.1 \mathrm{mM}$ IPTG, and $1 \mathrm{mM}$ IPTG). GFP fluorescence was quantified as a function of distance from the wall of the channel at positions indicated by the arrowheads, and is plotted to the right. Fluorescence values were normalized by setting the maximum fluorescence to 1. (d) Fluorescence detection of arabinose-regulated GFP expression under conditions identical to (c); the corresponding normalized fluorescence values are plotted to the right. (e) Detection of Anl incorporation by labeling with TAMRA-alkyne dye. Conditions are identical to those used for (c). The plot to the right shows the TAMRA fluorescence intensity (red) as a function of distance from the wall of the channel. TAMRA fluorescence values represent the experimental output of the AND gate, and were normalized by setting the maximum fluorescence to 1 . Black dots represent the products of the GFP fluorescence signals from plots in (c) and (d), which were point-wise multiplied to provide a measure of the predicted output of the AND gate. The products were normalized by setting the maximum signal to 1 in order to allow direct comparison to the TAMRA fluorescence values.

constructs that expressed GFP under control of the same $\mathrm{P}_{\mathrm{T}}$ and $\mathrm{P}_{\mathrm{BAD}}$ promoters that were used for expression of the $\mathrm{N}$ and C-terminal fragments of the AND gate. GFP expression revealed the activation profile of each promoter as a function of position across the width of the channel (Figure $4 c-e$ ).

Cells harboring the AND gate were immobilized in the microfluidic channel, exposed to the inducer gradients and Anl for $4 \mathrm{~h}$, then fixed and treated with an alkyne-functionalized AlexaFluor 488 dye. Fluorescence imaging showed that Anl 
incorporation was confined to cells in the center of the channel (Figure 4e).

To confirm that the observed labeling profile is predictable from the strengths of the input signals, we used the activation profile of each promoter as reported by GFP expression. As expected, either for a bimolecular binding equilibrium or for a Boolean AND operation, the scalar product of the inputs closely matched the output signal as measured by Anl incorporation (Figure 4e). This result suggests that the protein labeling profile can be adjusted, by tuning the input promoter strengths, to be more- or less-sharply dependent on the gradients of local activators or morphogens. Conversely, using endogenous promoters to drive the AND gate should provide labeling outputs that closely match spatial patterns of promoter activation.

This work introduces a new AND gate based on protein fragment complementation. ${ }^{6}$ The AND gate can be used to restrict protein labeling to subsets of cells in which specified promoters or genes of interest are active. Because the AND gate is genetically encoded, it should be easily integrated with previously reported transcription-based logic gates (e.g., AND, OR, NOR, and XOR gates) $)^{15,18,19}$ to create more complex genetic circuits with protein labeling as the output. As shown previously, ${ }^{1,20,21}$ azide-labeled proteins can be affinity-tagged and purified for analysis by mass spectrometry. Thus the AND gate provides a powerful new tool for targeted analysis of cellular protein synthesis.

\section{ASSOCIATED CONTENT}

\section{S Supporting Information}

Experimental details and additional information. This material is available free of charge via the Internet at http://pubs.acs.org.

\section{AUTHOR INFORMATION}

\section{Corresponding Author}

tirrell@caltech.edu

\section{Present Addresses}

${ }^{\#}$ Genomatica Inc., San Diego, CA 92121

${ }^{\infty}$ Massachusetts Institute of Technology, Cambridge, MA 02139

${ }^{\mathbb{I}}$ Princeton University, Princeton, NJ 08540

\section{Notes}

The authors declare no competing financial interest.

\section{ACKNOWLEDGMENTS}

We are grateful for financial support by National Institutes of Health grant NIH RO1 GM062523 and the Programmable Molecular Technology Initiative of the Gordon and Betty Moore Foundation (D.A.T.), the Institute for Collaborative Biotechnologies through grant W911NF-09-0001 from U.S. Army Research Office (D.A.T. and R.F.I.), the American Heart Association (J.J.S.), and the Robert A. Welch Foundation (J.J.S.). A.M. was supported by a scholarship from the National Science and Engineering Research Council of Canada and by a postgraduate scholarship from the Donna and Benjamin M. Rosen Center for Bioengineering at Caltech.

\section{REFERENCES}

(1) Dieterich, D. C.; Link, A. J.; Graumann, J.; Tirrell, D. A.; Schuman, E. M. Proc. Natl. Acad. Sci. U.S.A 2006, 103, 9482.
(2) Ngo, J. T.; Champion, J. A.; Mahdavi, A.; Tanrikulu, I. C.; Beatty, K. E.; Connor, R. E.; Yoo, T. H.; Dieterich, D. C.; Schuman, E. M.; Tirrell, D. A. Nat. Chem. Biol. 2009, 5, 715.

(3) Ngo, J. T.; Babin, B. M.; Champion, J. A.; Schuman, E. M.; Tirrell, D. A. ACS. Chem. Biol. 2012, 7, 1326.

(4) Rostovtsev, V. V.; Green, L. G.; Fokin, V. V.; Sharpless, K. B. Angew. Chem., Int. Ed. 2002, 41, 2596.

(5) Nakanishi, K.; Ogiso, Y.; Nakama, T.; Fukai, S.; Nureki, O. Nat. Struct. Mol. Biol. 2005, 12, 931.

(6) Ullmann, A.; Jacob, F.; Monod, J. J. Mol. Biol. 1967, 24, 339.

(7) Burbaum, J. J.; Schimmel, P. Biochemistry 1991, 30, 319.

(8) Segall-Shapiro, T. H.; Nguyen, P. Q.; Dos Santos, E. D.; Subedi, S.; Judd, J.; Suh, J.; Silberg, J. J. J. Mol. Biol. 2011, 406, 135.

(9) Mellot, P.; Mechulam, Y.; Le Corre, D.; Blanquet, S.; Fayat, G. J. Mol. Biol. 1989, 208, 429.

(10) Somerville, C. R.; Ahmed, A. J. Mol. Biol. 1977, 111, 77.

(11) Schmitt, E.; Tanrikulu, I. C.; Yoo, T. H.; Panvert, M.; Tirrell, D. A.; Mechulam, Y. J. Mol. Biol. 2009, 394, 843.

(12) Tanrikulu, I. C.; Schmitt, E.; Mechulam, Y.; Goddard, W. A., III; Tirrell, D. A. Proc. Natl. Acad. Sci. U.S.A 2009, 106, 15285.

(13) Tornoe, C. W.; Christensen, C.; Meldal, M. J. Org. Chem. 2002, 67, 3057.

(14) Hong, V.; Presolski, S. I.; Ma, C.; Finn, M. G. Angew. Chem., Int. Ed. 2009, 48, 9879 .

(15) Wang, B.; Kitney, R. I.; Joly, N.; Buck, M. Nat. Commun. 2011, 2,508 .

(16) Lucchetta, E. M.; Lee, J. H.; Fu, L. A.; Patel, N. H.; Ismagilov, R. F. Nature 2005, 434, 1134.

(17) Berk, V.; Fong, J. C.; Dempsey, G. T.; Develioglu, O. N.; Zhuang, X.; Liphardt, J.; Yildiz, F. H.; Chu, S. Science 2012, 337, 236.

(18) Tamsir, A.; Tabor, J. J.; Voigt, C. A. Nature 2011, 469, 212.

(19) Moon, T. S.; Lou, C.; Tamsir, A.; Stanton, B. C.; Voigt, C. A. Nature 2012, 491, 249.

(20) Hodas, J. J.; Nehring, A.; Hoche, N.; Sweredoski, M. J.; Pielot, R.; Hess, S.; Tirrell, D. A.; Dieterich, D. C.; Schuman, E. M. Proteomics 2012, 12, 2464.

(21) Eichelbaum, K.; Winter, M.; Diaz, M. B.; Herzig, S.; Krijgsveld, J. Nat. Biotechnol. 2012, 30, 984. 